\title{
La prévision hydrométéorologique et la conduite en crue des ouvrages EDF de production d'électricité : l'exemple des crues de février et mai 1999 sur le Rhin
}

\author{
The hydrometeorological forecast and the management of EDF electricity \\ production structures in case of flood : the example of the floods of \\ February and May 1999 on the Rhine River
}

par Ch. Lallement, EDF-DTG, Grenoble

R. Hirth, J. Schittly, EDF - Energie EST, Mulhouse

Whether flash on small mountainous catchments or slower on some big hydrographical basins, the flood is a major concern for the safety management of electricity production structures.

To face it up, EDF has set up an integrated system of supervision and hydrometeorological forecast which allows to alert the dam operators early enough and brings them a precious assistance to come to a decision.

We describe the system and its organisation, from the field measurement and the hydrological forecast to the reach of decision and the experimental returns.

The recent event of the Rhine's flood of February and May 1999 is taken as an example.

After a description of the phenomenons, of its follow-up, of the ensuing experiment returns, we show what such a supervision brings to the right management of dams in terms of operation safety, floods control and navigation.

\section{INTRODUCTION}

La crue, en tant que facteur d'aléa, est un souci majeur pour la conduite en sécurité des ouvrages de production d'électricité. Pour y faire face, EDF a mis en place un système intégré de surveillance et de prévision hydrométéorologique qui permet la mise en alerte des exploitants suffisamment à l'avance et leur apporte une aide précieuse dans leurs prises de décision.

Dans le présent article, on présente successivement : - les moyens de surveillance et de prévision des crues qu'Electricité de France a progressivement mis en place,

- le cas spécifique des aménagements du Rhin, illustré par les deux crues récentes de février puis mai 1999.

Il paraît utile de rappeler en préalable que la gestion des aménagements hydroélectriques en période de crue est une activité délicate à organiser, et ceci pour au moins trois raisons essentielles :

- c'est d'abord une activité qui ne peut être planifiée longtemps à l'avance, les crues étant fonction d'éléments naturels, échappant au contrôle humain,

- d'autre part, la faible occurrence des situations extrêmes susceptibles de conduire à des accidents majeurs permet difficilement de vérifier à l'échelle de temps humaine l'efficacité des dispositions adoptées,
- enfin, la diversité des aménagements exploités par EDF empêche d'édicter des principes d'organisation valables partout et par suite mal adaptés à des conditions locales.

Une gestion des crues mal maîtrisée sur un ouvrage pourrait aggraver, voire provoquer des inondations en amont et en aval, aux conséquences dommageables ; qui plus est, les crues augmentent sensiblement les risques d'atteinte à la sûreté intrinsèque de l'ouvrage en sollicitant, aux limites de leurs capacités, les divers organes constitutifs et en particulier ceux concourant à l'évacuation des débits.

C'est pourquoi, l'exploitant d'ouvrages hydroélectriques poursuivra durant un épisode de crues deux objectifs essentiels :

- empêcher la submersion de l'ouvrage, afin de maintenir son intégrité : une étude statistique des accidents de barrage montre en effet que le risque majeur de rupture résulte de leur submersion ; pour les grands réservoirs, la rupture provoquerait une onde de submersion aux conséquences bien plus catastrophiques que celles de la crue.

- assurer la transparence de l'ouvrage, i.e. que les conséquences de la crue ne seront pas aggravées par la présence de l'ouvrage. En pratique, cela se traduit par le fait que : - en aval, le débit maximal en sortie du barrage relevé lors de l'épisode de crue ne dépassera pas le débit maximal entrant, 
- pour les barrages mobiles en rivière, les berges et les terrains en amont immédiat de la retenue ne seront pas submergés du seul fait de la présence de l'ouvrage.

\section{1 LES PRINCIPES DE LA SURVEIL- LANCE DES CRUES À EDF}

\subsection{Préambule}

Pour satisfaire ses obligations de gestion des ouvrages en crue, EDF a installé depuis plus de 20 ans - sur les aménagements importants de tête de vallée (Rhône, Ain, Arc de Maurienne, Isère, Durance, Chassezac, Loire, Rhin, Dordogne, Tarn, Truyère...) - des systèmes de surveillance opérationnelle des crues qui apportent un peu d'anticipation sur les montées d'eau et sont un élément d'information précieux pour l'exploitant hydroélectrique.

Outils d'aide à la décision, ces systèmes restent toutefois découplés des moyens de conduite et ne sauraient servir à piloter directement des organes ou des ouvrages.

Ces systèmes de surveillance reposent sur quatre idées force que l'on détaille ci-après :

- une veille météorologique permanente, permettant l'identification et l'anticipation des situations à risque,

- des réseaux d'observations au sol, permettant de suivre la formation et le déroulement du phénomène de crue, - des outils de modélisation hydrologique, permettant de quantifier les évolutions à court terme des débits,

- des démarches systématiques de retour d'expérience, permettant d'ajuster la performance de l'ensemble au juste besoin, dans un contexte de maîtrise des coûts.

L'ensemble fait appel à une organisation, des personnels, des équipements hautement spécialisés et dévolus spécifiquement à ces tâches.

\subsection{Une première étape : la prévision météorolo- gique}

Une première étape de la surveillance des crues consiste à mener un suivi permanent de la situation météorologique.

Pour gagner en effet en anticipation sur les crues, il est nécessaire de remonter à la genèse du phénomène et de s'intéresser - en les analysant finement et en prévoyant leur évolution - aux situations météorologiques qui peuvent les engendrer.

Il s'agit d'analyser, au jour le jour, la situation météorologique à l'échelle de toute l'Europe et d'apprécier en termes de risque la probabilité d'apparition d'une situation hydrométéorologique dangereuse. En France, ce travail d'analyse de la situation météorologique est normalement dévolu à Météo France. A EDF cependant, tant pour des raisons historiques que techniques ou économiques, une structure intermédiaire totalement opérationnelle et dotée de moyens techniques importants a été mise en place en interface de Météo France. Cette structure est constituée de deux Centres Hydrométéorologiques régionaux respectivement situés à Grenoble et à Toulouse.

Grâce aux outils qu'EDF s'est constitués en propre (recherche automatique de situations analogues, base de connaissance pour le prévisionniste, ...), ou qui sont mis à disposition par Météo France (cartes météorologiques observées et prévues à échéances de 6 à 72 heures (modèle ARPEGE),
36 à 144 heures (modèle Centre Européen de Readings), imageries radar et satellitaire, visualisation temps réel des impacts de foudre, ...), le prévisionniste est en mesure, à tout moment, d'identifier toute situation "à risques".

\subsection{Une seconde étape : les observations de terrain}

Cette veille hydrométéorologique permet de bien croiser les prévisions et les informations météorologiques avec les conditions hydrologiques qui prévalent sur le terrain.

En effet, la surveillance des crues ne peut se construire qu'à partir d'un réseau de mesure au sol contrôlant les paramètres essentiels de la genèse des crues : précipitations, enneigement, températures de l'air, débits de rivière.

EDF, à cette fin, a créé et exploite un réseau de mesures de plus de 1200 points sur l'ensemble du territoire français. Ce réseau de mesures constitue un investissement à long terme mené depuis plus de 40 ans et un capital, accumulé au fil des ans, sous forme de fichiers de données numériques, historiques, contrôlés et critiqués.

L'ensemble de ce réseau fait l'objet de visites périodiques de la part d'équipes spécialisées en hydroclimatologie. Ces équipes assurent l'entretien et le contrôle des appareils en place, la vérification de leur étalonnage ainsi que des observations complémentaires ponctuelles. La moitié de ce parc de stations, soit 600 points, est télétransmise par téléphone, ou pour les points les plus sensibles, par satellite.

L'ensemble de ces stations délivre des mesures horaires, qui sont collectées au moins une fois par jour, plus fréquemment en périodes de crues, et qui sont mises à disposition des exploitants sur des serveurs informatiques implantés dans les installations EDF de tête de vallée.

\subsection{Les outils de modélisation hydrologique}

La prévision des débits s'effectue ensuite par des modèles mathématiques globaux ; on distingue deux grandes familles de modélisations, adaptées à la dynamique de la formation des crues sur le bassin surveillé.

\subsubsection{Les modèles de type pluiel débit}

Sur les petits bassins, la seule observation des débits peut ne pas être suffisante pour pouvoir disposer d'une véritable avance sur la crue. On remonte donc, pour disposer d'un peu plus de marge, le plus en amont possible dans la genèse du phénomène, notamment vers les pluies génératrices. La prévision des évolutions de débits s'effectue par un modèle mathématique de type pluie/débit qui admet en entrée, une donnée unique - la pluie moyenne qui tombe sur le bassin pendant un pas de temps choisi - et en sortie, la suite des débits à venir à l'exutoire du bassin considéré.

Une des difficultés réside dans la détermination de cette précipitation moyenne sur le bassin surveillé, surtout sur les bassins à forte hétérogénéité spatiale des précipitations.

L'adaptation et l'optimisation de la densité des réseaux sol d'observation de la pluviométrie reste, encore aujourd'hui, la meilleure réponse opérationnelle à cette question. L'utilisation, en complément, du radar météorologique pour les zones à fort gradient pluviométrique (Cévennes, bordure méditerranéeene, Corse, Pyrénées orientales, ...) est une piste qu'il convient d'explorer. Mais les outils et les techniques disponibles demandent encore à évoluer. 
Une autre difficulté tient à la bonne prise en compte des facteurs d'initialisation avant une crue notamment en ce qui concerne l'état de saturation des sols.

\subsubsection{Les modèles de type débit/débit}

Pour des bassins de taille supérieure, où les crues sont déjà formées sur les parties amont, on cherche davantage à étudier la propagation de l'onde de crue et la combinaison de crues sur des affluents divers. La prévision des évolutions de débits s'effectue par des modélisations de type débit/débit qui admet, en entrée, des données de débit (ou de leurs variations sur un pas de temps choisi) sur un ou plusieurs sites amont du bassin et, en sortie, la variation de débit à venir à l'exutoire du bassin considéré. Les principales difficultés résident alors dans la prise en compte d'apports intermédiaires variables entre les entrants amont et le point de prévision aval et dans l'évolutivité des fonctions de propagation en fonction du débit. Le recours à des techniques avancées d'identification permet, dans de nombreux cas, de lever ces difficultés et de cerner, avec la précision requise, les prévisions de débits qui sont utiles pour l'exploitant.

Pour ces deux familles de modèles, les observations permanentes de débits aux points stratégiques permettent d'affiner ou de rectifier la prévision effectuée.

\subsection{Les démarches systématiques de retour d'expérience}

Toutes ces informations - météorologiques et hydrologiques, simples observations ou prévisions - doivent être considérées comme un tout dont chaque élément, souvent indissociable ou complémentaire d'un autre, n'est là que pour aider les prévisionnistes à mieux percevoir l'ensemble de la situation et à l'appréhender en termes de risque. C'est ainsi que l'imagerie satellitaire permet de mieux localiser les masses nuageuses à fort développement vertical, qui sont celles susceptibles de produire de fortes précipitations, mais n'a vraiment d'intérêt en prévisionnel que couplée avec des cartes météorologiques observées et prévues.

Reste aussi que ces informations sont parfois porteuses de certitudes trompeuses et qu'elles nécessitent un vrai savoirfaire pour leur interprétation utile.

Après chaque épisode, le comportement de l'ensemble du système est analysé tant en termes "hydrologiques" (étude du phénomène) qu'en termes de "tenue des appareils de terrain" (fiabilité des réseaux de mesure et de télécommunication), de "fonctionnement des équipes" (qualité de l'organisation, respect des procédures), de "performance" des outils de prévision (pertinence des modélisations), de "qualité finale" de la surveillance (satisfaction de l'exploitant EDF). Ces démarches de retour d'expérience pilotent l'ensemble de l'activité, du choix des types de capteur sur le terrain jusqu'à l'adaptation des organisations, qui sont placées sous régime d'astreinte depuis septembre 1996.

\section{LE CAS PARTICULIER DU RHIN}

On décrit rapidement ci-après le retour d'expérience réalisé à l'issue des crues du Rhin de février puis mai 1999, ce dernier épisode constituant l'événement hydrologique le plus fort connu depuis la mise en service de l'usine de Kembs en 1932.

\section{- 3.1. Caractéristiques des aménagements du Rhin, de Bâle à Lauterbourg}

A l'entrée en France à Bâle, les débits caractéristiques du Rhin sont les suivants :

- Débit moyen :

$1060 \mathrm{~m}^{3} / \mathrm{s}$

- Débit d'étiage :

$350 \mathrm{~m}^{3} / \mathrm{s}$

- Débit extrême

$6000 \mathrm{~m}^{3} / \mathrm{s}$

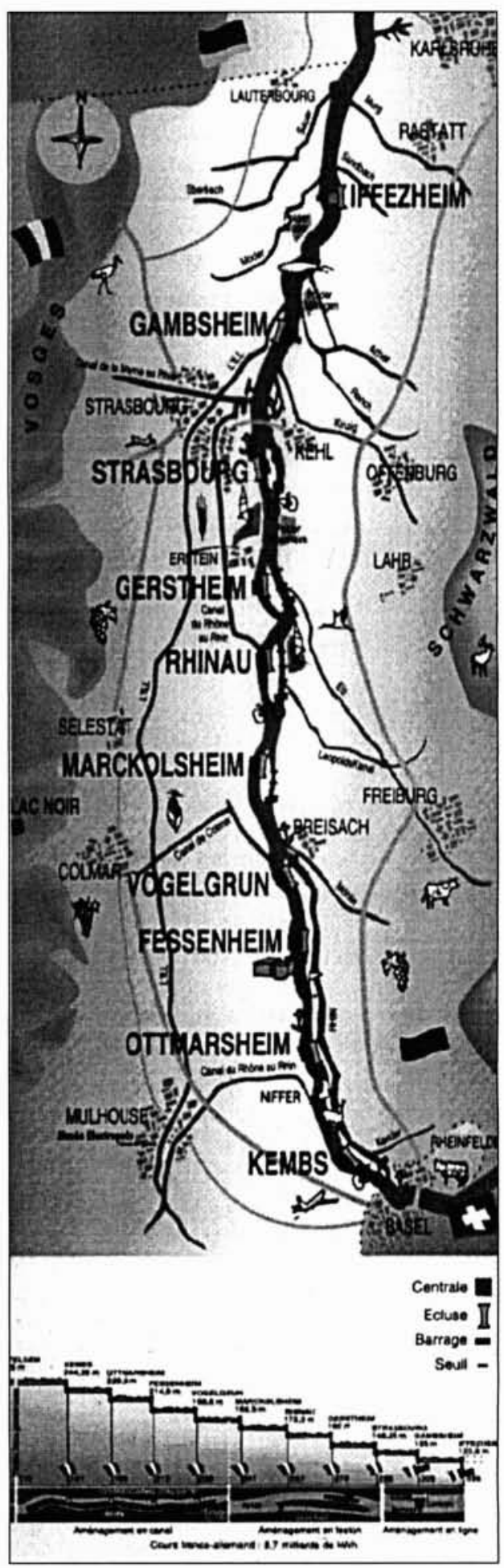

1. Le Rhin de Bâle à Lauterbourg. 
Le tronçon franco-allemand du Rhin s'étire sur $183 \mathrm{~km}$ pour un dénivelé de $133 \mathrm{~m}$. Il comporte aujourd'hui 10 chutes successives représentant une puissance électrique de $1400 \mathrm{MW}$ et une production de 8,5 milliards de $\mathrm{kWh}$ par an. Son trafic fluvial est d'environ 25 millions de tonnes et de 30000 bateaux par an à hauteur de Strasbourg.

L'aménagement des chutes a comporté 3 phases :

- $1^{\text {ère }}$ phase : 1932-1959

Mise en service des 4 premières chutes sur le Grand Canal d'Alsace parallèle au Rhin : Kembs, Ottmarsheim, Fessenheim et Vogelgrun avec un barrage en tête, à Kembs à quelques km à l'aval de Bâle.

- $2^{\text {ème }}$ phase : 1961-1970

Mise en service des 4 chutes suivantes de Marckolsheim, Rhinau, Gerstheim, et Strasbourg. Ces chutes, dites "en feston", comportent chacune un barrage et un canal de dérivation.

- $3^{\text {ème }}$ phase : 1974-1978

Mise en service des 2 dernières chutes de Gambsheim et d'Iffezheim. Ces aménagements ont été réalisés dans un cadre franco-allemand sur une base de 50/50 (convention franco-allemande de 1969) contrairement aux 8 premières chutes qui sont purement françaises (basées sur le traité de Versailles et la convention franco-allemande de 1956). Pour ces 2 chutes, l'ensemble des ouvrages (barrage mobile, digue de fermeture, centrale et écluses) sont alignés sur un même axe transversal.

\subsection{Exploitation du Rhin en période de crue}

Au-delà du débit d'équipement des chutes $\left(1400 \mathrm{~m}^{3} / \mathrm{s}\right)$, la régulation des débits et niveaux des biefs est assurée par les calculateurs des barrages, télésurveillée par le poste central de conduite de la chaîne d'aménagements installée à Kembs (Poste Hydraulique de Vallée = PHV). A noter qu'à Kembs, existe une loi d'abaissement du niveau au droit du barrage pour limiter la remontée du niveau d'eau dans le port de Bâle.

En 1982, la France et l'Allemagne ont signé une convention prévoyant la réalisation d'un important programme de protection contre les crues visant à retrouver une situation équivalente à celle d'avant l'aménagement du fleuve. Une des mesures importantes de cet accord concerne les manœuvres exceptionnelles des usines EDF.

Des consignes franco-allemandes prévoient, lors des crues exceptionnelles et sur la base de différents critères de débits et de leur évolution, l'engagement de manœuvres de basculement progressif de l'essentiel du débit turbiné vers le barrage. Ainsi, en fin de ces manœuvres exceptionnelles, pratiquement tout le débit du fleuve s'écoule au droit des barrages dans l'ancien lit du fleuve. L'effet d'écrêtement du débit est ainsi obtenu par écrêtement naturel des eaux (effet retard et effet de stockage supplémentaire). Ces manœuvres concernent actuellement les centrales sur le Grand Canal d'Alsace et celles de Marckolsheim, Rhinau et Gerstheim.

Bien évidemment, ces manœuvres entraînent des pertes de production importantes (plusieurs millions de francs par manæuvre) d'où l'importance de la pertinence de leur mise en œuvre dans les cas limites. La difficulté principale pour l'exploitant est liée justement à ces cas limites où l'on tangente parfois assez longuement les critères de mise en œuvre prévus dans les consignes. Dans ces conditions, la connaissance des prévisions d'évolution des débits à court terme et leur fiabilité est absolument primordiale pour la prise de décision (engagement, attente ou demande de dérogation). Les responsabilités juridiques et économiques attachées à ces décisions sont ainsi très fortes.

\section{- 3.3. La surveillance hydrométéorologique effectuée sur le Rhin}

Les principes généraux de la surveillance des crues à EDF s'appliquent entièrement pour le Rhin à Kembs, qui présente néanmoins les spécificités suivantes :

- la totalité du bassin versant se trouve en Suisse ou en Allemagne,

- la nature et la taille de ce bassin versant (36 $494 \mathrm{~km}^{2}$ ) sont telles que les crues se forment en aval du système des grands lacs Suisses (Bodensee, Zugersee, Vierwaldstättersee, etc.).

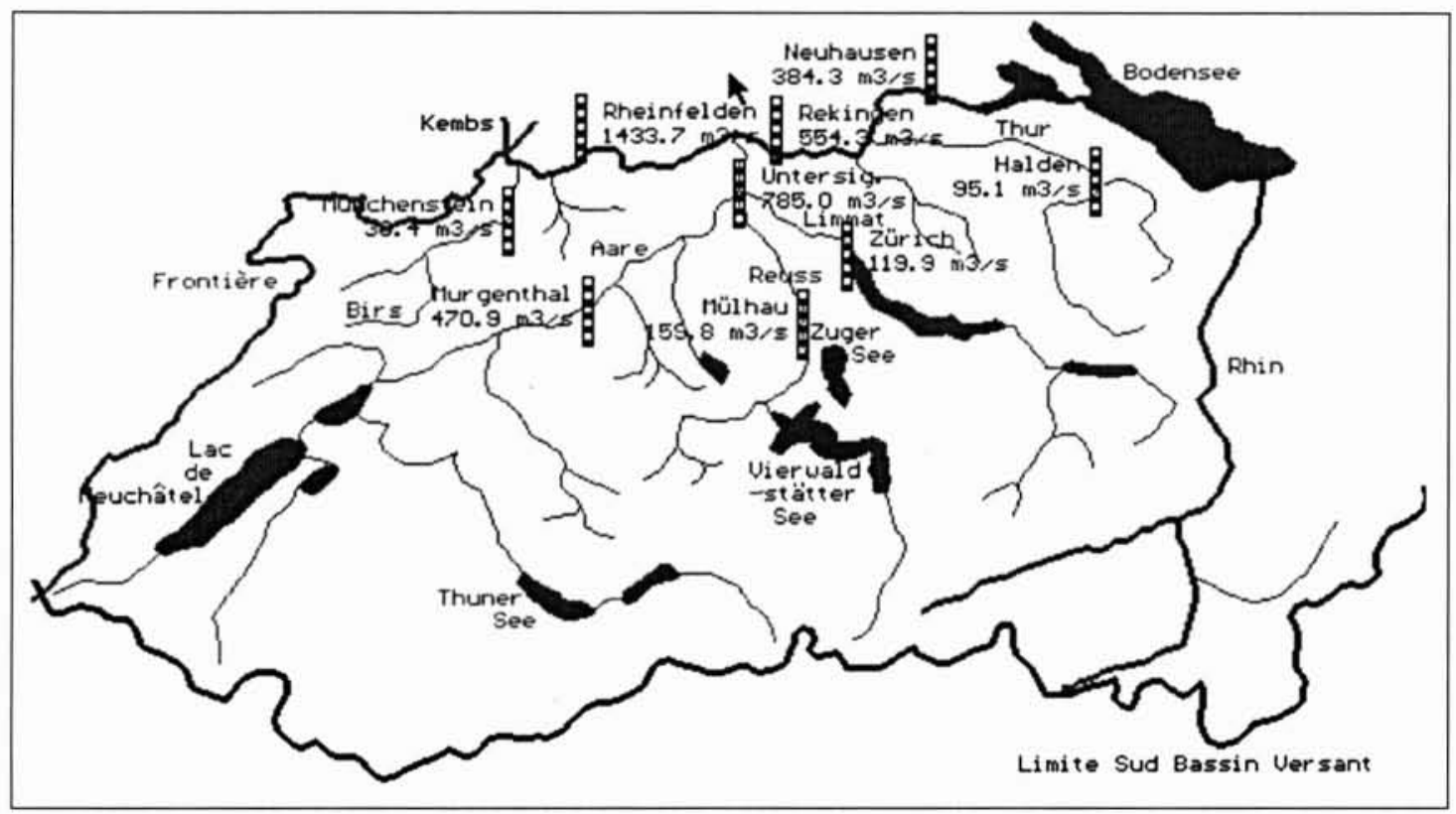

2. Carte du bassin du Rhin à Kembs. 


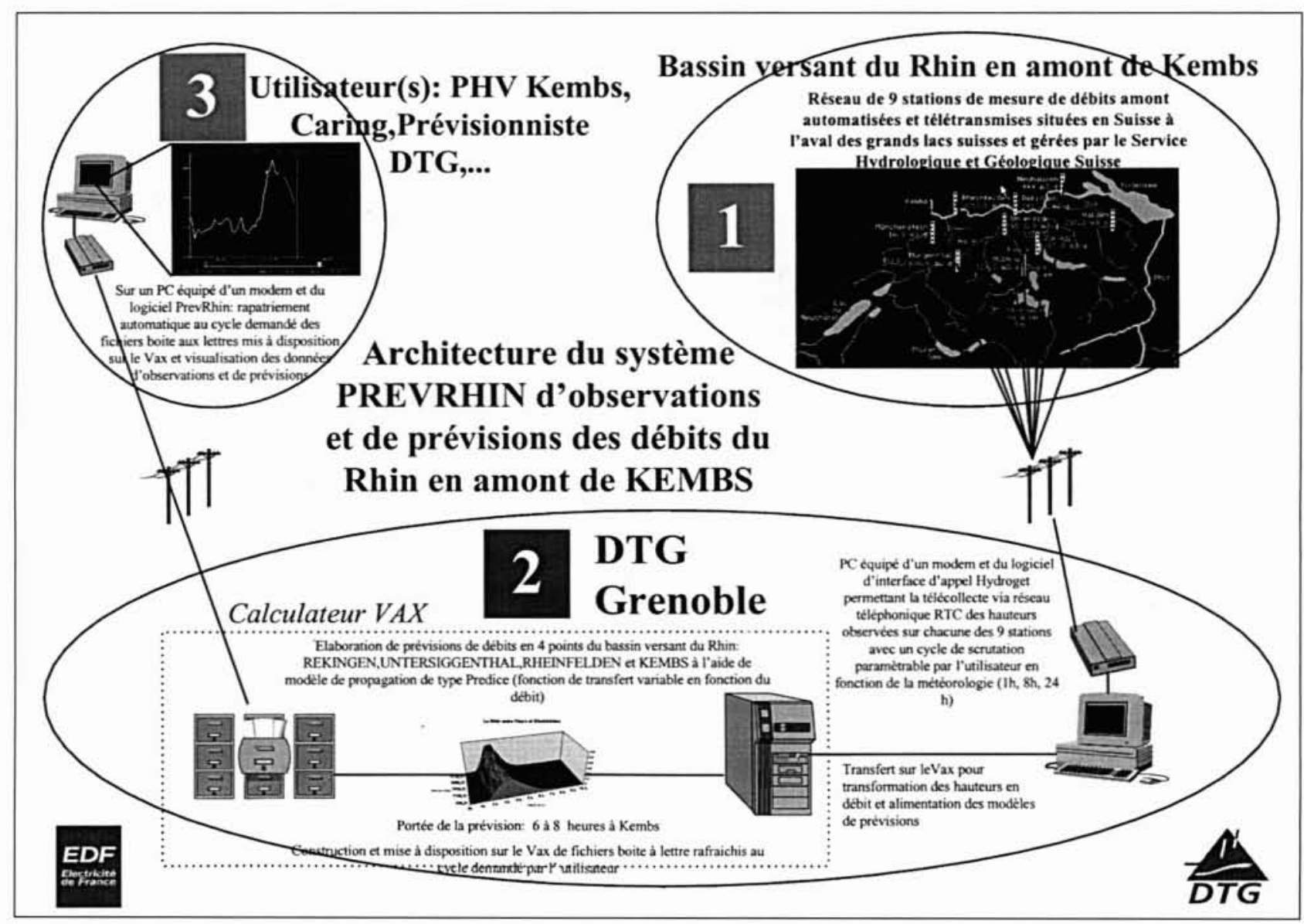

3. Synoptique du système de prévision des crues du Rhin à Kembs.

C'est pourquoi, EDF a engagé des opérations de surveillance des crues du Rhin depuis de nombreuses années, basées sur l'interrogation de témoins hydrométriques suisses. Après plusieurs phases d'évolution (1992...), le système a été complètement modernisé en 1998 ; avec l'accord de l'organisme compétent, les données de neuf stations hydrométriques du Service Hydrologique Fédéral Suisse sont télécollectées directement par EDF sur les capteurs et mémoires statiques en place. Des modèles de prévision hydrologique ensuite mis en œuvre sont de type débit/débit et consistent en la combinaison de trois modèles de propagation emboîtés (Rhin amont Aare, branche Aare, Rhin aval Aare). Ils se distinguent du modèle de prévision du Service Hydrologique Fédéral Suisse, :

- par sa conception, le modèle suisse reposant sur une combinaison de modèles pluie/débit puis débit/débit qui nécessitent en données d'entrée supplémentaires des observations et des prévisions quantitatives de précipitation, - par son caractère opérationnel, fonctionnant chaque heure, tandis que le modèle suisse - plus lourd à mettre en œuvre - n'a ses prévisions rafraîchies qu'une fois par jour.

Les informations d'observations et de prévisions ainsi constituées par EDF sont en outre mises à disposition, via un serveur informatique de données, au Poste Hydraulique de Vallée du Rhin à Kembs et au Centre d'Alerte Rhénan et d'Informations Nautiques de Gambsheim (CARING) chargé de l'annonce des crues sur la partie française du Rhin, comme outil d'aide à la décision.

On présente figures 2 et 3 une carte du bassin du Rhin à
Kembs, puis un synoptique qui détaille l'architecture du système de surveillance des crues en amont de Kembs.

\subsection{La crue du Rhin de Février 1999}

\subsubsection{Le phénomène hydrologique}

Un régime perturbé de Nord-Ouest à Ouest s'installe sur la moitié Nord de la France et la Suisse du 17 au 23 février 1999. Trois perturbations océaniques successives sont à l'origine d'importants cumuls de précipitations (plus de $200 \mathrm{~mm}$ en 5 jours sur le Jura). Une limite pluie/neige oscillant entre $400 \mathrm{~m}$ (le 18) et $1400 \mathrm{~m}$ (le 22) amène un lessivage partiel du manteau neigeux fraîchement constitué, qui contribue fortement à l'amplification des crues.

Partis d'un niveau relativement bas à $640 \mathrm{~m}^{3} / \mathrm{s}$ le 18 février, les débits du Rhin montent tout au long du 19 puis du 20 pour atteindre une première pointe de crue le 20 février vers $23 \mathrm{~h}$. Une accalmie relative s'installe jusqu'au passage d'une seconde pointe de crue le 22 février vers $12 \mathrm{~h}$ à $3600 \mathrm{~m}^{3} / \mathrm{s}$. La circulation atmosphérique ensuite orientée au Nord permet une descente d'air froid et instable ; la limite pluie neige redescend en plaine et entraîne la décrue.

Cette crue constituait la plus forte réalisation connue sur le Rhin de ces dernières années, depuis le Printemps 1994.

\subsubsection{Le suivi réalisé}

Le chronogramme de la figure 4 montre l'enchainement des messages émis par le Centre de Prévision vers le Poste Hydraulique de Vallée du Rhin à Kembs. On note les éléments saillants suivants : 
CRUE DU RHIN DU 22 FEVRIER 1999 : synthèse du suivi et des prévisions effectués par le Centre Hydrométéorologique Alpes pour le compte du PHV Kembs

Contact téléphonique exploitants

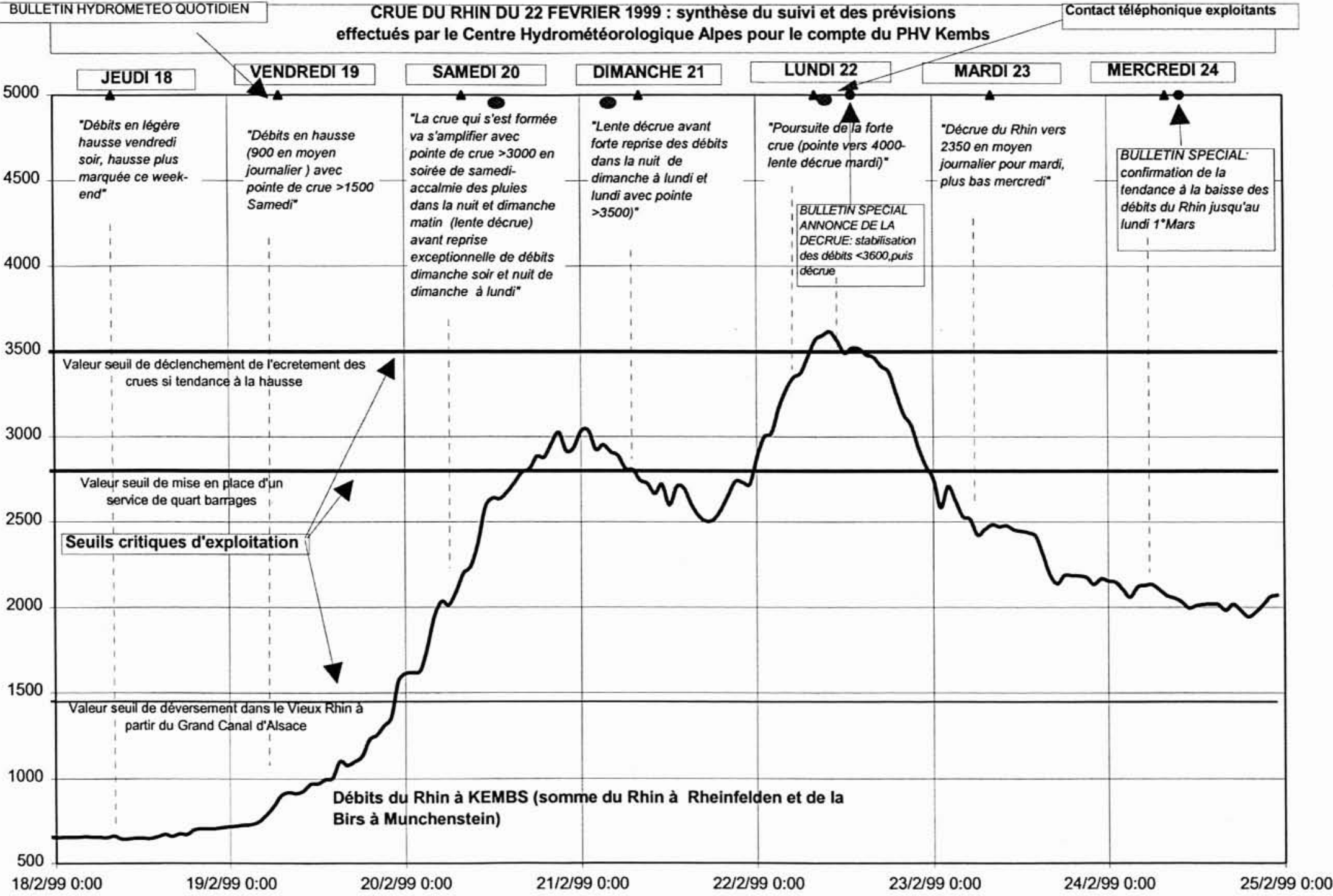

4. Crue du Rhin du 22 février 1999 : synthèse du suivi et des prévisions. 
1) la situation de crue est détectée en matinée du jeudi 18 février 1999,

2) le vendredi 19 février à $9 \mathrm{~h} 30$, un bulletin annonce le franchissement probable du seuil de $1500 \mathrm{~m}^{3} / \mathrm{s}$ avec 12 heures d'anticipation,

3) le samedi 20 février à $10 \mathrm{~h} 30$, un bulletin annonce le franchissement probable du seuil de $3000 \mathrm{~m}^{3} / \mathrm{s}$ avec 14 heures d'anticipation ; une décrue est prévue pour le 21 . avant une reprise exceptionnelle des débits,

4) le dimanche 21 février à $10 \mathrm{~h} 20$, un bulletin annonce un dépassement de $3500 \mathrm{~m}^{3} / \mathrm{s}$ pour le 22 , précédé d'une lente décrue toute la journée du 21 ,

5) le lundi 22 février à 9 h30, annonce d'une crue à $4000 \mathrm{~m}^{3} / \mathrm{s}$ en soirée ; en fait le Rhin plafonne à $3600 \mathrm{~m}^{3} / \mathrm{s}$ dès $14 \mathrm{~h} 40$. Un bulletin spécial vers $16 \mathrm{~h}$ confirme la décrue qui s'installe sur plusieurs jours.

\subsubsection{Les problèmes rencontrés}

Le système d'interrogation des stations suisses s'est arrêté brutalement à deux reprises occasionnant l'absence de données temps réel durant quelques heures (le 21 février entre 4 et $7 \mathrm{~h}$; le 22 février à $16 \mathrm{~h}$ ). Ces lacunes momentanées d'information n'ont pas affecté le suivi, mais ont permis d'identifier un défaut logiciel sur les protocoles d'appel des stations hydrométriques, résolu depuis.

Un retour d'expérience effectué a posteriori sur le modèle de prévision montre que celui-ci a présenté une certaine inertie : de comportement correct dans les phases intermédiaires de montée puis de décrue, il sous-estime les débits en début de crue, puis surestime les pointes de crue à l'approche de celles-ci. C'est ce qui a été à l'origine de la prévision surestimée à $4000 \mathrm{~m}^{3} / \mathrm{s}$ en matinée du 22 . L'explication proposée relie cette inertie de comportement au rôle particulier joué par le lessivage du manteau neigeux au cours de l'épisode, rendant atypique cette crue du 22 février 1999 par rapport à l'échantillon de crues qui a servi au calage des paramètres du modèle.

\subsection{La crue du Rhin de mai 1999}

\subsubsection{Le phénomène hydrologique}

Un printemps tardif mais estival vaut à partir de fin Avril des températures élevées sur l'ensemble des Alpes, supérieures aux normales d'un mois de juin. Ce temps doux entraîne une fusion soutenue de l'important manteau neigeux qui s'est constitué cet hiver sur les Alpes Suisses, plus forte réalisation d'enneigement de ces vingt dernières années, guère entamé jusque là par le temps maussade.

Sur ces abondants débits de base ( $>2000 \mathrm{~m}^{3} / \mathrm{s}$ ), un courant de Sud Ouest humide et doux s'établit sur la Suisse du 11 au 17 mai ; il provoque une série de violentes pluies orageuses du 10 au 12 mai (cumuls de l'ordre de 70 à $150 \mathrm{~mm}$; intensités horaires maximales de 37 à $65 \mathrm{~mm} / \mathrm{h}$ ) accompagnées d'une réplique moins active le 14 mai au matin (20 à $60 \mathrm{~mm}$ ). L'isotherme $0^{\circ} \mathrm{C}$ avoisinant $2800 \mathrm{~m}$ durant tout l'épisode, $95 \%$ du bassin du Rhin reçoit ainsi des précipitations sous forme liquide ; le lessivage du manteau neigeux en place aggrave les débits résultant des seules précipitations liquides.

1) Les débits du Rhin montent très rapidement dans la nuit du 11 au 12 mai, pour atteindre $4970 \mathrm{~m}^{3} / \mathrm{s}$ à Kembs le 13 mai à 0 h00,

2) Une décrue des tributaires rive gauche du bassin s'engage qui ramène le Rhin à $4455 \mathrm{~m}^{3} / \mathrm{s}$ à Kembs vers
10 h00 le 13 mai ; cette décrue correspond à l'accalmie du temps dans l'après-midi du 12 mai,

3) mais la reprise des averses en soirée du 12 mai freine cette décrue et en conséquence, les débits remontent jusqu'à $4560 \mathrm{~m}^{3} / \mathrm{s}$ à Kembs le 13 mai à $15 \mathrm{~h} 00$.

4) la décrue se confirme ensuite jusqu'au lendemain (14 mai) 5 h00, pour être interrompue par une reprise des débits suite au deuxième passage perturbé dans la nuit du 13 au 14 mai. Les débits remonteront jusqu'à $3960 \mathrm{~m}^{3} / \mathrm{s}$ le 14 mai à $20 \mathrm{~h} 00$.

La décrue se confirmera ensuite bénéficiant d'un temps plus calme. Toutefois un régime moyen de $2500 \mathrm{~m}^{3} / \mathrm{s}$ sera ensuite entretenu jusqu'au début du mois de juin par une série de perturbations alimentant régulièrement le bassin versant.

Cette crue constitue la troisième plus forte réalisation connue depuis 1808 sur le Rhin à Bâle, après juin 1876 $\left(5700 \mathrm{~m}^{3} / \mathrm{s}\right)$ et septembre $1881\left(5280 \mathrm{~m}^{3} / \mathrm{s}\right)$.

\subsubsection{Le suivi réalisé}

Le chronogramme de la figure 5 montre l'enchaînement des messages émis par le Centre de Prévision vers le Poste Hydraulique de Vallée du Rhin à Kembs. On note les éléments saillants suivants :

1) la situation de crue est détectée en matinée du mercredi 12 mai 1999 ,

2) le mercredi 12 mai à 21 h09, un bulletin consigne par écrit les prévisions orales de $17 \mathrm{~h} 00$, elles-mêmes confirmées par le modèle de prévision hydrologique,

3) le jeudi 13 mai à $9 \mathrm{~h} 37$, un bulletin spécial confirme les prévisions orales émises vers $7 \mathrm{~h}$ d'une possibilité de dépasser à nouveau $5000 \mathrm{~m}^{3} / \mathrm{s}$ en début d'après-midi du 13 mai, et une ultime crue dans la nuit du 14 au 15 mai puis le retour au calme pour les 15 et 16 mai,

4) le jeudi 13 mai vers $12 \mathrm{~h} 50$, un nouvel échange est réalisé avec le Service Hydrologique Fédéral Suisse, dont les prévisions de débit ne sont pas en accord avec les prévisions EDF, 5) le jeudi 13 mai à $19 \mathrm{~h} 46$, le bulletin précise que de nouvelles pluies sont en route pour le bassin du Rhin dont l'influence se fera sentir vers $10 \mathrm{~h}$ le 14 mai à Kembs ; ce nouvel épisode sur un débit de base d'environ $3300 \mathrm{~m}^{3} / \mathrm{s}$ devrait engendrer des débits supérieurs à $4000 \mathrm{~m}^{3} / \mathrm{s}$ sans dépasser les débits de la veille. Le débit passera sous les $3000 \mathrm{~m}^{3} / \mathrm{s}$ au plus tard le samedi 15 au soir.

6) le vendredi 14 à $10 \mathrm{~h} 45$, le bulletin spécial annonce que la dernière vague orageuse est en cours et amènera les débits à $4000 \mathrm{~m}^{3} / \mathrm{s}$ en fin d'après-midi pour ensuite aborder la décrue avec moins de $3000 \mathrm{~m}^{3} / \mathrm{s}$ le samedi après-midi. 7) le vendredi 14 à $15 \mathrm{~h} 45$, le bulletin prévoit $4000 \mathrm{~m}^{3} / \mathrm{s}$ en soirée et un retard de la décrue due à une réactivation de la perturbation sur le haut bassin de l'Aare.

8) le samedi 15 mai à $8 \mathrm{~h} 12$, la décrue du Rhin qui a commencé à $6 \mathrm{~h} 00$ va se confirmer, on attend $3600 \mathrm{~m}^{3} / \mathrm{s}$ à $12 \mathrm{~h} 00$ et $3200 \mathrm{~m}^{3} / \mathrm{s}$ à $24 \mathrm{~h} 00$.

\subsubsection{Les problèmes rencontrés}

Le capteur de la station hydrométrique de Neuhausen (contrôle de la sortie du Bodensee) est rapidement submergé par les eaux du Rhin ; en conséquence, la liaison est interrompue avec le point de mesure pour toute la durée de l'épisode.

Durant la nuit du 12 au 13 mai, le grand nombre d'appels émis par les différents acteurs ont rendu inaccessibles l'ensemble des stations hydrométriques pendant une fenêtre de 2 heures. 
CRUE DU RHIN DU 13 MA 1999 : synthése du suivi et des prévisions effectués par le Centre Hydrométéorologique Alpes pour le compte du PHV Kembs

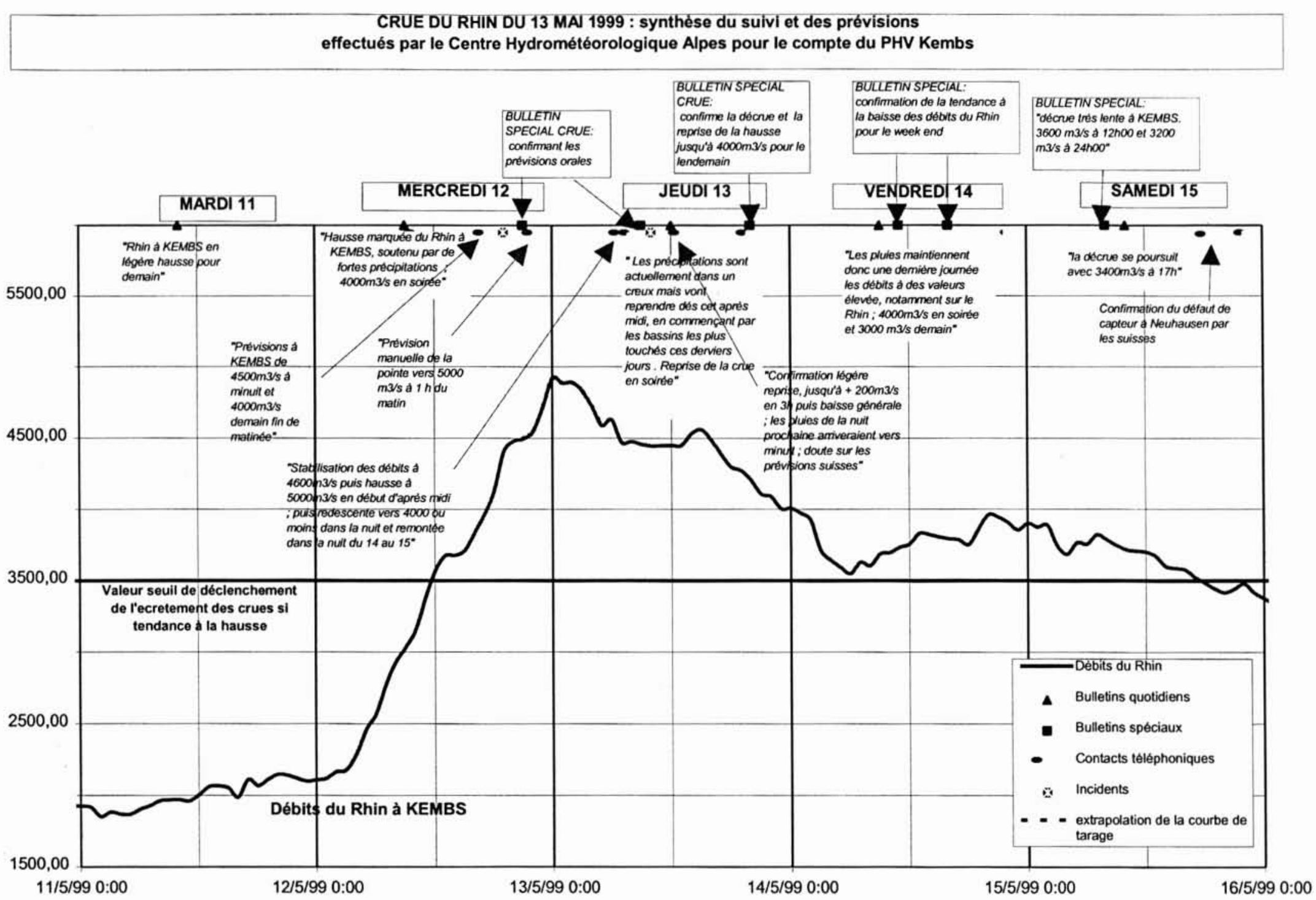




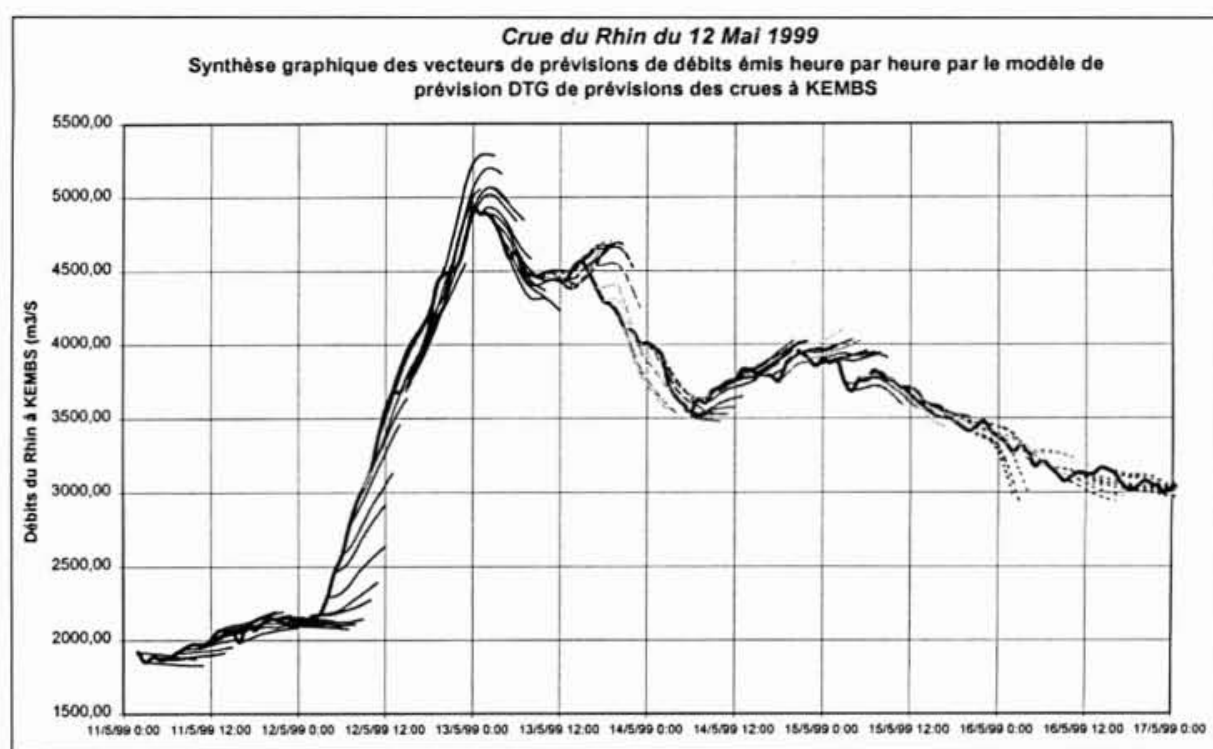

6. Crue du Rhin du 12 mai 1999.

Les cotes atteintes sont telles que dès l'après-midi du 12 mai, les débits ne sont plus calculés sur les stations hydrométriques amont (cotes "hors barème"). En conséquence, le modèle de prévision des crues du Rhin ne pouvait plus fonctionner de façon automatique. Le prévisionniste d'astreinte prolonge rapidement les courbes de tarage et fait fonctionner le modèle de prévision "sur un coin de table", de façon dégradée. Les débits de pointe sont annoncés à mieux que $10 \%$ près et pour l'heure $h$.

Un retour d'expérience effectué a posteriori sur le modèle de prévision montre que son comportement est resté très satisfaisant, tant sur les phases de crue que de décrue.

\subsection{L'utilisation de la surveillance hydrométéorolo- gique par les exploitants d'ouvrage}

S'agissant de faire un retour d'expérience de l'utilisation de la surveillance hydrométéorologique par les exploitants des ouvrages hydroélectriques du Rhin, on relèvera les quatre points essentiels ci-après :

La surveillance hydrométéorologique réalisée a permis l'activation rapide d'une cellule de crise, et ceci tant en février qu'en mai 1999, dès le début de l'événement.

La qualité des prévisions émises a facilité ensuite les prises de décision ; la bonne anticipation des phénomènes a permis d'atténuer l'effet de surprise et ceci dans un contexte aggravé par le fait que les aménagements du Rhin faisaient face au plus grand événement hydrologique de leur existence, avec des débits roulés qui atteignaient plus de 5 fois l'écoulement moyen couramment constaté.

Sur le Rhin, lors de chaque crue importante, s'établit une concertation permanente entre les cellules de crises française (EDF, CARING), allemande (HVZ) et suisse (RDS de Bâle). Les décisions des uns dépendant des prévisions des autres ou ayant une incidence forte chez les autres, il est très important que les experts disposent d'un maximum d'informations fiables permettant si possible des recoupements pertinents. Dans le cas précis des crues de février et mai 1999, EDF a, à plusieurs reprises, fondé sa position sur les prévisions hydrométéorologiques de la DTG en opposition parfois avec l'avis d'experts externes.
Le retour d'expérience a montré qu'ainsi, EDF a pu prendre, au cours de ces épisodes de crues, les bonnes décisions au bon moment et trouver le bon compromis entre sa responsabilité d'exploitant et les sollicitations externes pour déroger ou non aux consignes en vigueur.

Une bonne appréhension des évolutions de débit dans les 6 à 10 heures à venir a permis en outre de mieux répondre aux sollicitations et questions externes. Même si la responsabilité de l'annonce des crues de Bâle à Lauterbourg échoit au CARING, le Poste Hydraulique de Vallée du Rhin à Kembs est continument interrogé par des tiers sur le régime actuel du Rhin et ses variations prochaines.

Enfin, la surveillance hydrométéorologique a facilité la mise en état d'alerte et l'organisation des équipes de travail sur place, en aidant à la bonne allocation des ressources humaines sur les ouvrages, dans les services de quart aux barrages et dans les centrales pour les opérations de dégrillage.

\section{IV - CONCLUSIONS}

Une bonne gestion des crues sur un ouvrage hydroélectrique, en tant qu'elle participe à la sûreté de l'exploitation, est "au cœur du métier" de l'hydraulicien. Les données de prévisions des crues facilitent cette gestion en anticipant la mise en place de cellules d'alerte, aidant les décisions tant organisationnelles que de bonne exécution des manœuvres, étayant la communication et la justification des décisions prises.

La surveillance hydrométéorologique et la prévision des crues font donc partie intégrante de ces démarches d'amélioration constante de la sûreté et de la maîtrise des exploitations hydroélectriques.

Néanmoins, une surveillance hydrométéorologique de qualité ne peut être obtenue que grâce à une exigence de professionnalisme sur l'ensemble des maillons constitutifs du système : veille météorologique constante pour bien identifier les situations à risques, données hydrologiques disponibles et de qualité, expertise des situations, retours d'expériences systématiques pour une bonne capitalisation du savoir-faire et identification des points faibles.

Dans le contexte particulier du Rhin, fleuve international, à l'hydrologie complexe, la modernisation du système EDF de prévision des crues du Rhin a connu sa première mise à l'épreuve "en vraie grandeur" lors des crues de février, puis mai 1999 - ce dernier épisode constituant la plus grande crue connue sur le Rhin depuis la mise en service de Kembs (1932).

L'ensemble a prouvé sa pertinence, en fournissant des anticipations et prévisions quantitatives de débit de bonne qualité, et a permis d'engranger des éléments d'amélioration utiles pour les crues prochaines. 